\title{
CT assessment of the correlation between clinical examination and bone involvement in oral malignant tumors
}

\section{Marco Antonio Portela \\ Albuquerque ${ }^{(a)}$ \\ Marcia Etsuko Kuruoshi(b) \\ Ilka Regina Souza Oliveira ${ }^{(c)}$ \\ Marcelo Gusmão Paraíso \\ Cavalcanti(d)}

(a) PhD student; (d) Professor - Department of Radiology, School of Dentistry, University of São Paulo, São Paulo, SP, Brazil.

(b) Radiologist, University Hospital, University of São Paulo, São Paulo, SP, Brazil.

(c) Professor, Department of Radiology, School of Medicine, University of São Paulo, São Paulo, SP, Brazil.
Corresponding author:

Marcelo Gusmão Paraíso Cavalcanti

Departamento de Radiologia, Faculdade de

Odontologia, Universidade de São Paulo

Av. Prof. Dr. Lineu Prestes, 2227, Cidade

Universitária

São Paulo - SP - Brazil

CEP: 05508-900

E-mail:mgpcaval@usp.br

\begin{abstract}
Oral cancers have a tendency to invade the surrounding bone structures, and this has a direct influence on the treatment management and on outcomes. The objective of this study was to correlate the clinical parameters (location, clinical presentation and TNM staging) of oral malignant tumors that can be associated with a potential of bone invasion and determine the accuracy of clinical examination to predict bone involvement, using computed tomography (CT). Twenty five patients, with oral malignant tumors were submitted to clinical and CT examinations. CT was considered the standard parameter to evaluate the presence of bone involvement. Clinical assessment of location, presentation form and TNM staging of the tumors were then compared to the CT findings in predicting bone involvement. Bone involvement was observed in $68 \%$ of the cases. It was predicted that tumors located in the retromolar trigone and hard palate, with a clinical aspect of infiltrative ulcer or nodule and classified in stage IV had a high potential to cause bone involvement. The clinical examination assessment of these tumors showed to be a valuable tool to predict bone invasion, with high sensitivity (82\%) and specificity $(87.5 \%)$, based on the results found in the CT images. No statistical significance was found between the $\mathrm{CT}$ and clinical examinations regarding bone involvement. The identification of some clinical parameters such as location, clinical presentation, and TNM stage, associated with a detailed clinical examination, was considered a valuable tool for the assessment of bone destruction by oral malignant tumors.
\end{abstract}

Descriptors: Oral cancer; Diagnosis, clinical; Tomography, X-ray computed. 


\section{Introduction}

Malignant tumors of the oral mucosa are mainly represented by squamous cell carcinoma, whose incidence in this site accounts for approximately 5\% of all cases of this disease in the human body. ${ }^{1,2}$ Oral malignancies are responsible for considerable morbidity and mortality; therefore, an accurate staging, which includes evaluation of bone involvement, is crucial for treatment planning and for improving survival rates. ${ }^{2}$

These tumors have a tendency to invade the surrounding bone structures, and this occurs in $12 \%$ to $88 \%$ of the cases. ${ }^{1-10}$ From a treatment perspective, it is crucial to determine the bone involvement caused by these tumors and, more importantly, the amplitude of bone involvement, since an underestimation may lead to a partial resection, resulting in local recurrence and potential metastasis. ${ }^{2}$ On the other hand, an overestimation of bone involvement can lead to an unnecessary bone resection, resulting in considerable cosmetic and functional defects. ${ }^{11,12}$

Clinical evaluation, which mainly includes direct inspection and palpation, is a very important tool to assess the presence of bone involvement in oral malignacies. ${ }^{4,13}$ On the other hand, several imaging modalities such as conventional radiography, ultrasonography, computed tomography, bone scanning and magnetic resonance imaging have been used to investigate the presence of bone involvement by oral cancers. ${ }^{2,6,10,11,14}$ All these methods seem to have specific limitations; however, the use of computed tomography (CT) in the preoperative assessment of malignant tumors seems to be the most valuable technique, because it shows soft and bone tissues in the same exam and has a high sensitivity and specificity for the assessment of bone destruction. ${ }^{1,6}$

The aim of the present study was to correlate clinical parameters, such as location, clinical presentation and TNM staging, that can be associated with a potential of bone infiltration, and also to determine the sensitivity and specificity of clinical examination for the assessment of bone invasion by oral malignant tumors.

\section{Material and Methods}

Twenty-five patients (17 male and 8 female) with oral malignant neoplasms were seen at our clinic of Oral Diagnosis from August 2003 to August 2004. Patient age ranged from 29 to 84 years (mean 57.8 years). Initially, all patients were subjected to a complete clinical examination, which was followed by a biopsy and a histopathologic analysis of the lesion. In order to detect bone invasion by malignant tumors upon clinical examination, the main clinical parameters used were inspection, palpation, and description of lesions. Stage assessment was made according to the criteria of the $6^{\text {th }}$ edition TNM staging system established by the American Joint Committee on Cancer. ${ }^{15}$ The histopathologic analyses revealed that 22 cases $(88 \%)$ were squamous cell carcinomas, $2(8 \%)$ were mucoepidermoid carcinomas, and 1 was an adult T-cell leukemia lymphoma.

Subsequently, the patients were submitted to CT examination (TCT-600 HQ, Toshiba Medical Systems Inc., Tustin, CA, USA), in order to evaluate the extension of the tumor and the presence of bone involvement. The imaging protocol, designed by our Department, included axial and coronal 2-mm thick slices with $2 \mathrm{~mm}$ of interval of reconstruction in $2 \mathrm{~mm}$ of table feed. CT examinations were performed after the intravenous (IV) administration of iodine non-ionic contrast (Iopamiron 300, Schering AG, São Paulo, SP, Brazil) that was infused immediately before starting scanning. The CT images were considered the standard parameter in our study. All images were interpreted in consensus by two radiologists with long experience in diagnosing malignant tumors with CT, using soft-tissue and bone-tissue windows. Clinical features such as location, clinical presentation and TNM staging of the tumors were then compared to the bone destruction findings obtained in the CT examination, which was used as gold standard (Figures 1 and 2). The accuracy, sensitivity and specificity of the clinical examination in predicting bone involvement by oral malignant tumors were also evaluated by comparing its results with the CT results. The study was previously approved by the ethics committee of our Institution. The statistical analysis was performed with Bioestat 3.0 software, using the Wilcoxon non-parametric test to determine the difference between the clinical 

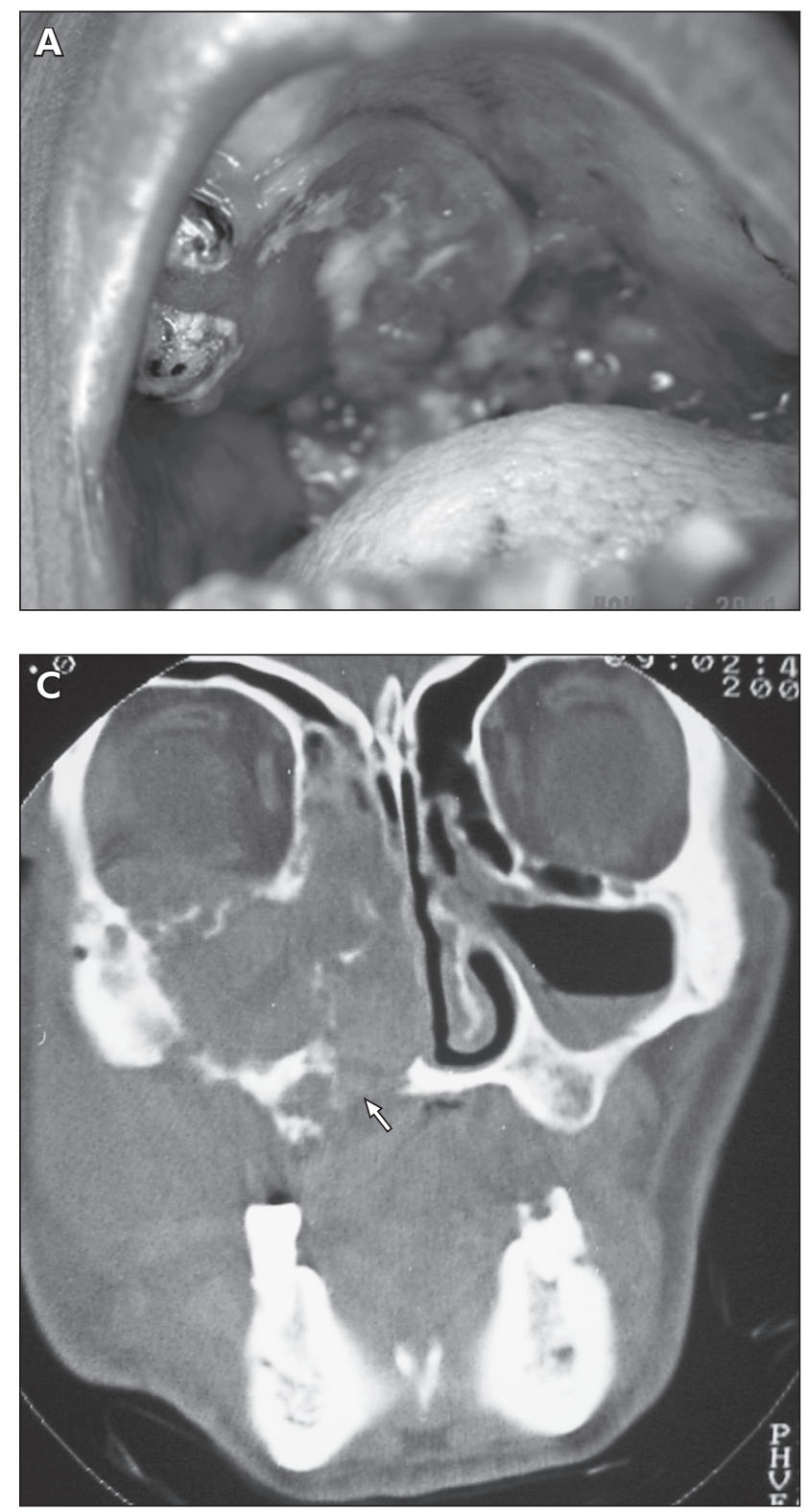

and CT examinations.

\section{Results}

\section{Correlation between clinical examination and $\mathrm{CT}$ images}

In the clinical examination, bone involvement was suspected in 15 cases $(60 \%)$, whereas CT detected the presence of bone involvement in 17 cases $(68 \%)$. Of the 15 cases with bone invasion detected by clinical examination, 1 was not confirmed by CT images, a false-positive case. Of the 10 cases with

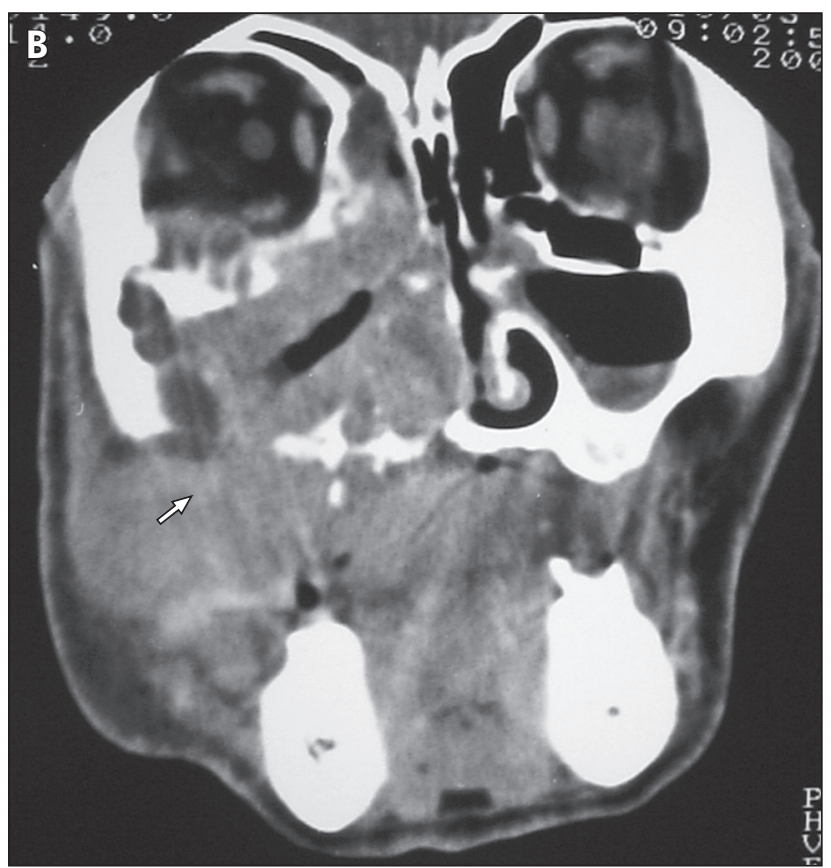

Figure 1 - These images show an infiltrative ulcer on the right hard palate (squamous cell carcinoma) extending to the oropharynx. The soft-tissue window coronal CT image shows a heterogeneous enhancement mass extending to the buccal space and superiorly to the maxillary and ethmoidal sinus, floor of the orbit and nasal cavity (B) (arrow). The bone window coronal CT image shows bone destruction of the floor of the maxillary sinus, orbit and lateral wall of the nasal cavity (C) (arrow). 

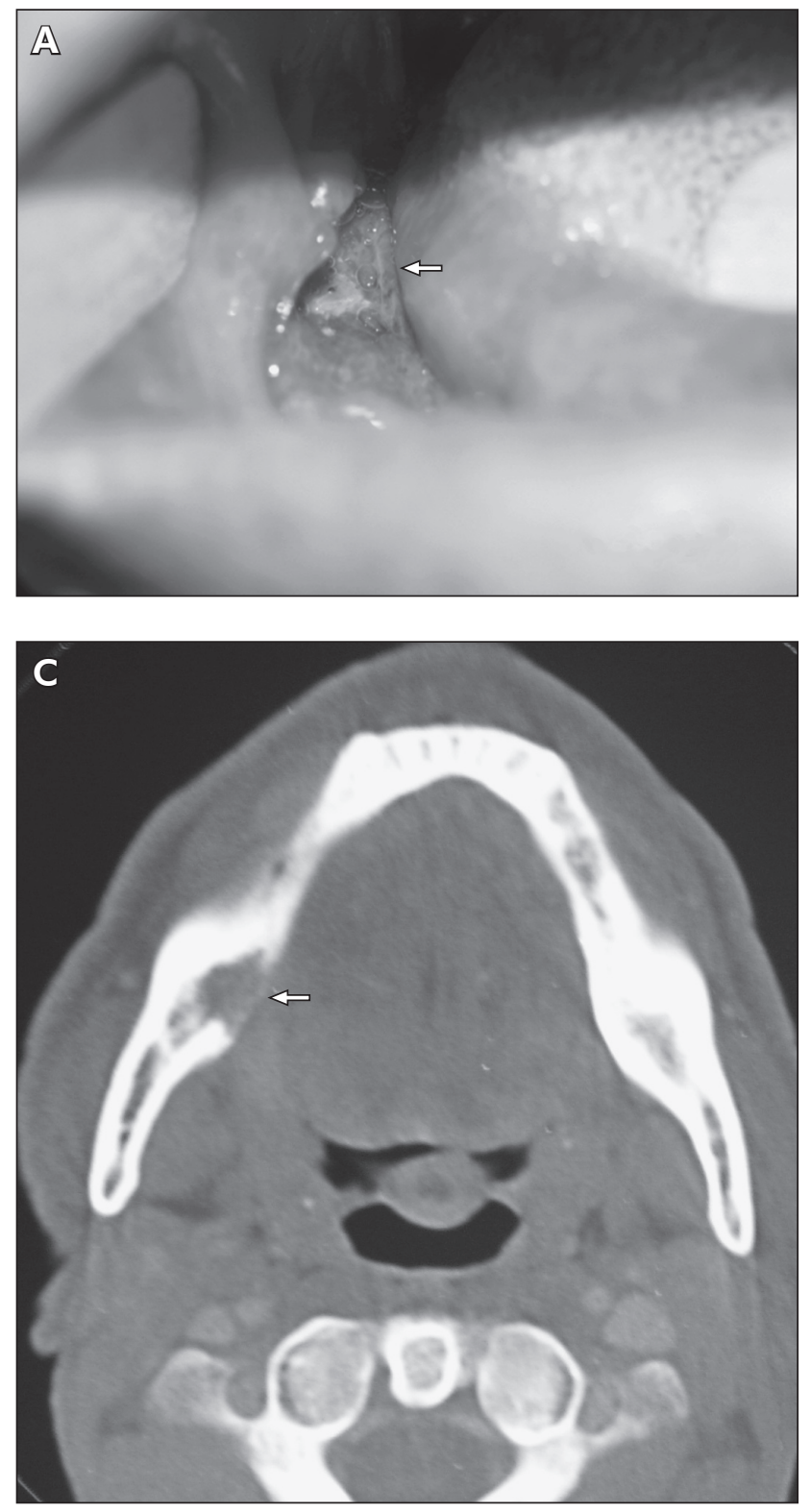

\section{Location}

Most of the tumors $(60 \%)$ were found in more than one anatomical structure, but only the initial location of the tumor was considered for the analysis. The floor of the mouth was the site most commonly affected, and was found in 7 patients $(28 \%)$. The retromolar trigone and hard palate were each involved in 4 cases $(16 \%)$, and gingiva and tongue were each involved in 5 patients $(20 \%)$. The correlation between the tumor site and the presence of bone involvement showed that lesions located in the retromolar trigone and hard palate caused bone

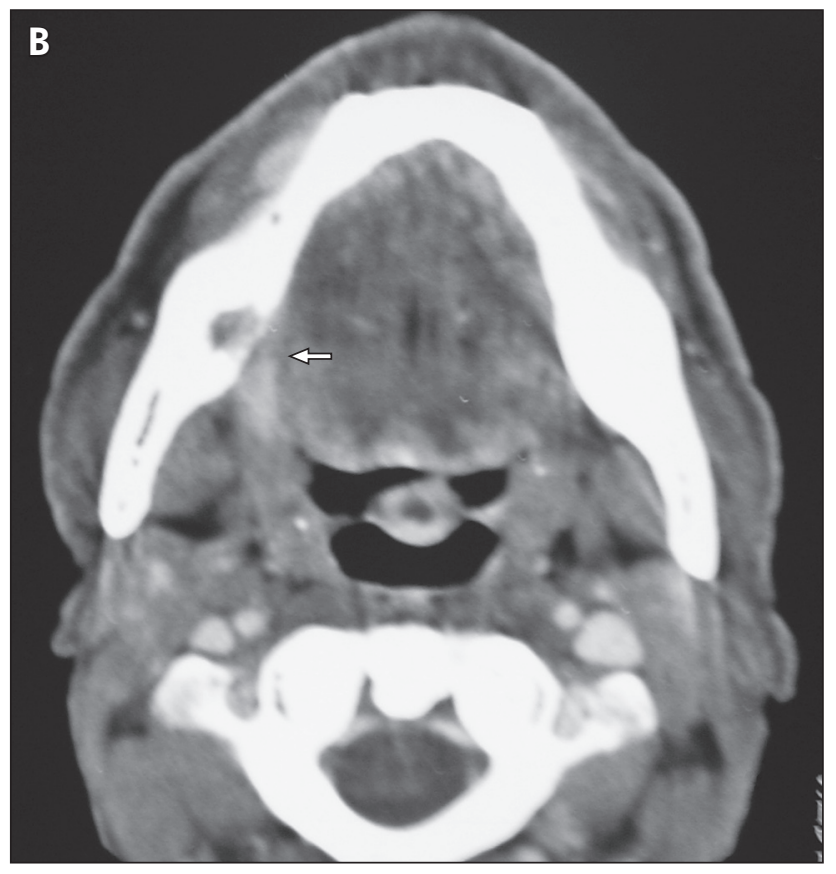

Figure 2 - These images demonstrated the clinical aspect of an infiltrative ulcer on the right retromolar trigone (squamous cell carcinoma). The soft-tissue window axial CT image shows a homogeneous enhancement mass on the floor of the mouth on the right side (B) (arrow). The bone window axial CT image shows buccal cortical bone destruction of the mandible on the right side (C) (arrow). destruction in $100 \%$ of the cases, while the lesions on the gingiva and on the floor of the mouth caused bone invasion, respectively, in $75 \%$ and $50 \%$ of the cases. None of the lesions located on the tongue produced bone invasion.

\section{Morphologic aspect of the lesions}

Sixteen patients presented lesions characterized by infiltrative ulcers, 6 had ulceration with papillary growth and 3 had nodular types. Bone involvement was seen in $100 \%$ of the nodular lesions whereas the infiltrative and papillary ulcers presented bone de- 
Table 1 - Assessment of the clinical exam sensitivity and specificity in the evaluation of bone destruction by oral and oropharyngeal tumors.

\begin{tabular}{c|c|c|c}
\hline \multirow{2}{*}{$\begin{array}{c}\text { Assessment of bone } \\
\text { destruction by clinical } \\
\text { examination }\end{array}$} & \multicolumn{2}{|c|}{$\begin{array}{c}\text { Assessment of bone } \\
\text { destruction by CT } \\
\text { - Gold Standard - }\end{array}$} & \multirow{2}{*}{ Total } \\
\cline { 2 - 3 } & Yes & No & \\
\hline Yes & 14 & 1 & 15 \\
\hline No & 3 & 7 & 10 \\
\hline Total & 17 & 8 & 25 \\
\hline
\end{tabular}

struction in $69 \%$ and $50 \%$ of the cases, respectively.

\section{Staging}

In order to analyze whether advanced lesions involved the bone more frequently than those in an initial stage, the stage assessment was determined with a view to correlating stage with tumor invasion into the bone. This relationship is presented in Table 2. It was observed that 21 of the 25 patients were classified in stage IV (84\%), 52\% in stage IVA and $32 \%$ in stage IVB. It was also observed that $76.2 \%$ of these 21 patients presented bone destruction caused by the tumor.

\section{Discussion}

Several imaging techniques, such as conventional radiography, CT, magnetic resonance and scintigraphy have been used to assess bone destruction by oral and maxillofacial lesions. ${ }^{2,3,5,16,17}$ CT scan appears to have specific advantages over any other technique for the evaluation of oral malignant neoplasms because it accurately shows soft tissue and bone structures in the same exam. Therefore, CT examination allows determining the extension of the lesion, regional node involvement as well as the presence of bone involvement. ${ }^{4}$

The reported incidence of bone involvement by tumors in the oral region varies from $12 \%$ to $88 \%$. $^{7-}$ 9,13 In our study, bone invasion was confirmed by CT in 17 cases (68\%), which was exactly the same rate as that reported by Kalavrezos et al. ${ }^{2}$ (1996). We analyzed tumors distributed in different sites of the oral region and oropharynx, and this may explain the high incidence of bone destruction found
Table 2 - Relationship between clinical stage according to the TNM system and bone destruction.

\begin{tabular}{|c|c|c|c|c|c|}
\hline \multirow{2}{*}{ Stage } & \multirow{2}{*}{ Criteria } & \multicolumn{4}{|c|}{ Bone invasion, number of patients } \\
\hline & & & No & Yes & Total \\
\hline । & TINO & & $(50 \%)$ & $1(50 \%)$ & 2 \\
\hline$\|$ & T2NO & & $100 \%)$ & 0 & 2 \\
\hline III & T2N1,T3N0,T3N1 & 0 & & 0 & 0 \\
\hline \multirow[t]{6}{*}{ IVA } & T4a NO MO & \multirow{6}{*}{\multicolumn{2}{|c|}{$3 \quad(23.1 \%)$}} & \multirow[t]{6}{*}{$10(76.9 \%)$} & \multirow[t]{6}{*}{13} \\
\hline & T4a N1 MO & & & & \\
\hline & T1 N2 MO & & & & \\
\hline & T2 N2 MO & & & & \\
\hline & T3 N2 MO & & & & \\
\hline & T4a N2 MO & & & & \\
\hline \multirow[t]{3}{*}{ IVB } & T4b Any N MO & \multirow{3}{*}{\multicolumn{2}{|c|}{$2(25 \%)$}} & \multirow[t]{3}{*}{$6(75 \%)$} & \multirow[t]{3}{*}{8} \\
\hline & Any T N3 M0 & & & & \\
\hline & IVC Any T Any N MI & & & & \\
\hline
\end{tabular}

Relative values in parentheses.

in our study.

While assessing clinical examination with regard to the presence of bone involvement by oral tumors, Close et al. ${ }^{3}$ (1986) evaluated 60 patients with tumors in the floor of the mouth, and stated that $53(88 \%)$ were correctly evaluated by clinical examination. Bimanual palpation of the floor of the mouth can provide information regarding adherence and infiltration of the cancer to the mandible. ${ }^{4}$ van den Brekel et al. ${ }^{17}$ (1998) also reported that clinical examination was an accurate (62\%) and a highly specific technique because it could easily predict the presence of bone infiltration. They reported no false-positive results in their study, and found a low sensitivity (39\%) and a high specificity $(100 \%)$. We found that, of the 25 patients assessed, the clinical examination correctly evaluated 21 cases, representing an accuracy of $84 \%$. This high accuracy rate was similar to that reported by Leipzig ${ }^{7}$ (1985). We observed that bone invasion was suspected clinically in 15 cases, and in 14 patients this involvement was confirmed by the CT. In addition, 10 of the 25 patients were considered to have no evidence of bone invasion by the clinical examination. However, three of these 10 patients had a CT confirmation of bone invasion, indicating a specific- 
ity of $87.5 \%$ and sensitivity of $82 \%$ for the clinical examination. The statistical analysis also confirmed no significant difference between the clinical and CT examinations.

The relationship between lesion site and presence of bone invasion has been analyzed by several studies. ${ }^{2,12}$ According to these studies, tumors located in the retromolar area and alveolar ridge are those most likely to invade bone ( $88 \%$ of the cases), since the soft tissue layer in these areas is quite thin, which allows a rapid bone invasion by the tumor. The other oral site where malignant tumors can invade the bone is the floor of the mouth, at a rate of $52 \% .^{2,18}$ We also correlated the sites of the tumors with the presence of bone destruction. We detected bone involvement in all of the lesions on the hard palate and retromolar trigone. Half of the lesions located on the floor of mouth had bone destruction, indicating results similar to those of previous studies. However, none of the lesions in the tongue presented bone invasion.

The clinical aspect of the tumors associated with the presence of bone involvement has not been reported previously by any other study. We found a close relation between these two parameters. Of the 16 patients presenting lesions with clinical aspect of an infiltrative ulcer, 11 (69\%) had bone invasion. This high propensity for bone invasion by these lesions might be due to the principal vector of growth toward the deep tissues. All of the 3 lesions with clinical aspect of a nodule also produced bone destruction. On the other hand, of the 6 patients with lesions with clinical appearance of a verrucous ulcer, only $3(50 \%)$ had bone involvement. The lesser propensity of verrucous ulcers to invade bone may be accounted for by the growth vector of these tumors, which is stronger in the direction opposite to the underlying tissues.

Tumor stage appears to be associated with mandibular bone involvement. This concept is supported by a study conducted by Brown et al. ${ }^{18}$ (1994), who examined 35 patients with oral carcinomas and observed that none of the patients in stages I and III had bone involvement. On the other hand, 2 out of 8 patients $(25 \%)$ in stage II, and 17 out of
19 patients $(89.4 \%)$ in stage IV had bone destruction. ${ }^{18}$ Kalavrezos et al. ${ }^{2}$ (1996) reported that only one patient out of 4 , in stage I, had evidence of bone invasion, and none of the patients with tumors in stage II had bone involvement. Tumors classified as being in stage III were found in 6 patients, and, in 4 of them $(66 \%)$, bone invasion was observed. Lesions in stage IV were found in 44 patients, and, in $36(81.8 \%)$, bone infiltration was observed. In our study, the new staging criteria for head and neck malignant tumors ( $6^{\text {th }}$ edition) was used according to the AJCC Cancer Staging Manual. ${ }^{15}$ We found that none of the patients with tumors in stages II and III had bone involvement. Of 2 patients with lesions in stage I, one had bone destruction. Twenty one tumors were classified as being in stage IV, 13 in stage IVA and 8 in stage IVB. Bone involvement was observed in $76.9 \%$ of the lesions in stage IVA and $75 \%$ of the lesions in stage IVB. These results indicate a high correlation between an advanced stage classification and bone involvement.

\section{Conclusion}

Assessment of clinical parameters appears to be a very useful method in the detection of bone involvement by oral malignant tumors. It was predicted that tumors located in the retromolar trigone and hard palate, with clinical aspect of infiltrative ulcer or nodule and classified in stage IV, had a high potential to present bone involvement. Hence, according to the results found in the CT examination, the assessment of the clinical examination proved to be a valuable tool to predict bone invasion by these tumors, with high sensitivity and specificity, although without statistical difference in comparison to the CT examination. It is therefore considered a valuable tool to assist the professional in establishing the therapeutic management of patients.

\section{Acknowledgements}

This study was supported by a grant from the CNPq (Marcelo Cavalcanti, \#309331/2003-0), and by financial aid from the CAPES (Marco A. P. Albuquerque). 


\section{References}

1. Cavalcanti MGP, Ruprecht A, Quets J. Progression of maxillofacial squamous cell carcinoma evaluated using computer graphics and spiral computed tomography. Dentomaxillofac Radiol. 1999;28(4):238-44.

2. Kalavrezos ND, Grätz KW, Sailer HF, Stahel WA. Correlation of imaging and clinical features in the assessment of mandibular invasion of oral carcinomas. Int J Oral Maxillofac Surg. 1996;25(6):439-45.

3. Close LG, Merkel M, Burns DK, Schaefer SD. Computed tomography in the assessment of mandibular invasion by intraoral carcinoma. Ann Otol Rhinol Laryngol. 1986;95(4):3838.

4. Gomez D, Faucher A, Picot V, Siberchicot F, Renaud-Salis JL, Bussières $\mathrm{E}$ et al. Outcome of squamous cell carcinoma of the gingiva: a follow-up study of 83 cases. J Craniomaxillofac Surg. 2000;28(6):331-5.

5. Hong SX, Cha LH, Lee EW, Kim J. Mandibular invasion of lower gingival carcinoma in the molar region: its clinical implications on surgical management. Int J Oral Maxillofac Surg. 2001;30(2):130-8.

6. Huntley TA, Busmanis I, Desmond P, Wiesenfeld D. Mandibular invasion by squamous cell carcinoma: a computed tomographic and histological study. Br J Oral Maxillofac Surg. 1996;34(1):69-74.

7. Leipzig B. Assessment of mandibular invasion by carcinoma. Cancer. 1985;56(5):1201-5.

8. Leslie A, Fyfe E, Guest P, Goddard P, Kabala JE. Staging of squamous cell carcinoma of the oral cavity and oropharynx: a comparison of MRI and CT in T- and N-staging. J Comput Assist Tomogr. 1999;23(1):43-9.

9. Millesi W, Prayer L, Helmer M, Gritzmann M. Diagnostic imaging of tumor invasion of the mandible. Int J Oral Maxillofac Surg. 1990;19(5):294-8.
10. Nakayama E, Yoshiura K, Yuasa K, Tabada O, Araki K, Sanda $\mathrm{S}$ et al. Detection of bone invasion by gingival carcinoma of the mandible: a comparison of intraoral and panoramic radiography and computed tomography. Dentomaxillofac Radiol. 1999;28(6):352-6.

11. Shaha AR. Preoperative evaluation of the mandible in patients with carcinoma of the floor of the mouth. Head Neck. 1991;13(5):398-402.

12. Smyth DA, O'Dwyer TP, Keane CO, Stack J. Predicting mandibular invasion in mouth cancer. Clin Otolaryngol Allied Sci. 1996;21(3):265-8.

13. Tsue TT, McCulloch TM, Girod DA, Couper DJ, Weymuller EA, Glenn M. Predictors of carcinomatous invasion of the mandible. Head Neck. 1994;16(2):116-26.

14. O’Brien CJ, Carter RL, Soo KC, Barr LC, Hamlyn PJ, Shaw $\mathrm{HJ}$. Invasion of the mandible by squamous carcinomas of the oral cavity and oropharynx. Head Neck Surg. 1986;8(4):24756.

15. American Joint Committee on Cancer (AJCC). Cancer staging manual. $6^{a}$ ed. Philadelphia: Lippincott-Raven publishers; 2002.

16. Bahadur S. Mandibular involvement in oral cancer. J Laryngol Otol. 1990;104(12):968-71.

17. van den Brekel MW, Runne RW, Smeele LE, Tiwari RM, Snow GB, Castelijns JA. Assessment of tumour invasion into the mandible: the value of different imaging techniques. Eur Radiol. 1998;8(9):1552-7.

18. Brown JS, Griffith JF, Phelps PD, Browne RM. A comparison of different imaging modalities and direct inspection after periosteal stripping in predicting the invasion of the mandible by oral squamous cell carcinoma. Br J Oral Maxillofac Surg. $1994 ; 32(6): 347-59$. 